\section{AB0099 TAXOL ALLEVIATES COLLAGEN-INDUCED ARTHRITIS IN MICE BY INHIBITING THE FORMATION OF MICROVESSELS}

J. Xu ${ }^{1}$, Z. Feng ${ }^{2}$, J. Zhu ${ }^{3}$, S. Chen ${ }^{1}$, X. Chen ${ }^{4}, \mathrm{~J} \mathrm{Li}^{3} .{ }^{1}$ Department of Internal Medicine of Traditional Chinese Medicine, College of Traditional Chinese Medicine, Southern Medical University, Guangzhou; ${ }^{2}$ Department of Traditional Chinese Medicine, Medical College of China Three Gorges University, Yichang; ${ }^{3}$ Department of Rheumatology, Nanfang Hospital, Southern Medical University: ${ }^{4}$ Department of Pathogen Biology, School of Public Health, Southern Medical University, Guangzhou, China

Background: Angiogenesis is one of the critical features in rheumatoid arthritis (RA). Uncontrolled neovascularization could help the infiltration of inflammatory cells and lead to synovial hyperplasia and bone destruction further. Precious studies have demonstrated that the medicine taxol (PTX) has anti-angiogenesis effects.

Objectives: To evaluate the inhibitory effects of PTX on angiogenesis in a collagen-induced arthritis (CIA) mouse model.

Methods: A total of 50 mice were used to induce a CIA mouse model with collagen II (CII) and complete Freund's adjuvant (CFA). Twenty-four with obvious arthritis syndrome were randomly divided into four groups: CIA model group, PTX $1.5 \mathrm{mg} / \mathrm{kg}$ group, PTX $1.0 \mathrm{mg} / \mathrm{kg}$ group, PTX $0.5 \mathrm{mg} / \mathrm{kg}$ group. In addition, 6 normal mice was regarded as the control group. PTX was administered by intraperitoneal injection in the PTX treatment groups 8 times every other day. Arthritis index scores, tissue pathology scores after HE staining and synovium microvessel density (MVD) analysis after immunohistochemical (IHC) staining were performed. Immunohistochemistry and ELISA were used to detect the expression of vascular endothelial growth factor (VEGF) and hypoxia-inducible factor- $\alpha$ (HIF-1 $\alpha$ ). Additionally, the correlation between MVD and pathological scores, level of VEGF and HIF-1 $\alpha$ in the synovium were also evaluated.

Results: After PTX treatment, the three intervention group arthritis index scores $(1.33 \pm 0.52,2.00 \pm 0.63,3.33 \pm 1.03)$ declined when compared with the CIA group $(5.67 \pm 1.03, p<0.001, p<0.001, p=0.016)$. The total histological scores in the three PTX treatment groups $(2.50 \pm 0.66,3.89 \pm 0.86,3.89 \pm 0.86)$ were lower than those in the CIA group $(7.67 \pm 0.79, p<0.001, p<0.001, p=0.007)$. Similarly, PTX significantly alleviated the scores for synovitis, pannus formation and bone destruction. Compared with the CIA group $\left(110.32 \pm 5.06 / \mathrm{mm}^{2}\right)$, the MVD of the three intervention groups decreased in dose-dependent manner $\left(17.05 \pm 1.97 / \mathrm{mm}^{2}, 34.73 \pm 2.36 / \mathrm{mm}^{2}, 57.55 \pm 2.72 / \mathrm{mm}^{2} ; \mathrm{p}<0.001\right.$, respectively). In addition, the expression of VEGF and HIF-1 $\alpha$ in synovial tissues and serum also decreased significantly after PTX treatment. Further analysis showed that MVD and pathological scores and levels of VEGF and HIF-1 $\alpha$ in the synovium were positively correlated $(r=0.921, r=0.944, r=0.889, r=0.969, r=0.933 ; p<0.001$, respectively).

Conclusions: PTX may alleviate CIA by suppressing angiogenesis, providing new insights into the treatment of RA. VEGF and HIF-1 $\alpha$ may be the target for PTX suppression of microvessel formation.

\section{References:}

[1] Smolen JS, Aletaha D, McInnes IB. Rheumatoid arthritis. The Lancet 2016.

[2] Park KS, Park MJ, Cho ML, et al. Type II collagen oral tolerance; mechanism and role in collagen-induced arthritis and rheumatoid arthritis. Mod Rheumatol 2009;19:581-9.

Disclosure of Interest: None declared

DOI: 10.1136/annrheumdis-2017-eular.2515

\section{AB0100 MTX COMBINED WITH CTX INHIBITED IL-6 INDUCED JAK2-STAT3 ACTIVATION AND THE INDUCTION OF P-GP IN PERIPHERAL BLOOD LYMPHOCYTES OF RHEUMATOID ARTHRITIS PATIENTS}

K. Chen ${ }^{1}$, Y. Mu ${ }^{1}$, K. Qin ${ }^{1}$, X. Zhao ${ }^{1}$, J. Dong ${ }^{1}$, X. $\mathrm{Li}^{1}$, C. Gao ${ }^{2}$, C. Wang ${ }^{1}$ ${ }^{1}$ Rheumatology, the Second Hospital of Shanxi Medical University, Taiyuan, China: ${ }^{2}$ Pathology, Joint Program in Transfusion Medicine, Brigham and Women's Hospital/Children's Hospital Boston, Harvard Medical School, Boston, United States

Background: Rheumatoid arthritis (RA) is a systemic autoimmune disease characterized by irreversible joint destruction and disability. At present, the biggest challenge in the field of RA treatment is the emergence of multidrug resistance (MDR) in the application of disease-modifying anti-rheumatic drugs (DMARDs).The Multidrug resistance Related Protein and Multi Drug Resistance protein 1 , also called P-glycoprotein can decreases the intracellular concentration of different drugs. Moreover, cytokines play an increasingly important role in the expression regulation mechanisms of P-gp, especially inflammatory cytokines IL6 . Previously, We have indicated that Interleukin-6 up-regulates P-gp in peripheral blood lymphocytes via the JAK2-STAT3 pathway in RA patients. Through more than ten-years' clinical research, we observed that the therapeutic cycle alliance of methotrexate (MTX) and cyclophosphamide (CTX) has had better curative effect and lower rate of adverse reaction in treating RA. And we didn't found the emergence of MDR. How can the therapeutic alliance of MTX and CTX overcome MDR in RA patients? This study will give the answer.

Objectives: To Clarify whether the therapeutic alliance of MTX and 4hydroperoxycyclophosphamide (4-HC) suppress expression and mRNA of P-gp in peripheral blood lymphocytes of RA through JAK2-STAT3 pathway.
Methods: RA patients without any DMARDs and biologic therapy $(n=15)$ were enrolled. P-gp expression level was detected by Flow Cytometry. P-gp mRNA of peripheral blood lymphocytes and the intracellular signaling pathway mediating the effects of MTX and 4-HC on IL-6-stimulated JAK2-STAT3 activation was assessed by RT-PCR.

Results: Compared with blank control group, IL-6 induced P-gp, JAK2 and STAT3 expression levels increased significantly $(P<0.05)$. Compared with IL-6 group, P-gp, JAK2 and STAT3 expression levels of 4-HC group and low MTX+4-HC group both decreased $(P<0.05)$. the expression levels of P-gp, JAK2 and STAT3 in low MTX group, middle MTX group and high MTX group were lower than IL-6 group, but there were no statistically significant differences $(P>0.05)$.

Conclusions: Our data indicated that MTX combined with CTX significantly inhibited IL-6 induced JAK2-STAT3 activation, as well as the induction of P-gp. Inhibition of IL-6-mediated multidrug resistance signaling pathways by the alliance of MTX and CTX may represents a new reversing drug-resistance therapeutic strategy for RA.

\section{References:}

[1] Li HX, Zhao W, Shi Y, et al. Retinoic acid amide inhibits JAK/STAT pathway in lung cancer which leads to apoptosis. Tumour Biol. 2015; 36(11): 8671-8678.

[2] Oh HM, Lee SW, Yun BR, et al. Vigna angularis inhibits IL-6-induced cellular signalling and ameliorates collagen-induced arthritis. Rheumatology (Oxford). 2014; 53(1): 56-64

[3] Li R, Cai L, Ren DY, Xie XF, Hu CM, Li J. Therapeutic effect of 7, 3'-dimethoxy hesperetin on adjuvant arthritis in rats through inhibiting JAK2-STAT3 signal pathway. Int Immunopharmacol. 2012; 14(2): 157-163.

[4] Yun M, Lee D, Park MN, et al. Cinnamaldehyde derivative (CB-PIC) sensitizes chemo-resistant cancer cells to drug-induced apoptosis via suppression of MDR1 and its upstream STAT3 and AKT signalling. Cell Physiol Biochem. 2015; 35(5): 1821-1830.

Disclosure of Interest: None declared

DOI: 10.1136/annrheumdis-2017-eular.5019

\section{AB0101 NEW MUTATED PEPTIDYLARGININE DEIMINASE FROM PORPHYROMONAS GINGIVALIS A TARGET IN EARLY RA CITRULLINATES MAJOR RA-AUTOANTIGENS}

M. Jenning ${ }^{1}$, B. Marklein ${ }^{1}$, J. Yetterberg ${ }^{2}$, G. Burmester ${ }^{1}$, K. Skriner $^{1}$ ${ }^{1}$ Department of Rheumatology and Clinical Immunology, Charité University Medicine, Berlin; ${ }^{2}$ Department of Rheumatology and Clinical Immunology, Karolinska Institutet, Stockholm, Germany

Objectives: Previous reports showed that peptidylarginine deiminase (PPAD) form Porphyromonas gingivalis (P.g.) is not able to citrullinate proteins internaly. New mutated PPAD (mPPAD) from P.g. involved in periodontal disease (PD) cloned out of $P . g$. strain was characterized and analyzed for its reactivity in sera from patients with systemic autoimmune diseases

Methods: We cloned a new enzymatically active recombinant mutated PPAD from P.g. MPPAD mutations and citrullination sites were analyzed by DNA sequencing and/or protein mass spectrometry. Autocitrullination activity it's enzymatic-activity and human autoantigen protein citrullination was investigated by 2D-Elektrophoresis, MS, immunoblot analysis and ELISA. Furthermore we tested anti-mPPAD/cit-mPPAD with human sera $(n=93)$ from early RA before and after onset of RA ( $n=30)$, established RA ( $n=32)$, SLE $(n=16)$ and healthy blood donors $(n=15)$ in ELISA assays. To study a potential impact on the RA mouse model (CAIA), mPPAD-containing vesicles from P.g. were injected by intraperitoneal injection (IP)

Results: Recombinant mPPAD lacks 43 amino acids at the N-terminus and exhibits so far two new amino acid mutations (amino acid position $73(\mathrm{~F}>\mathrm{L})$ and $447(E>V)$. We were able to demonstrate, mPPAD is enzymatically active over a huge $\mathrm{pH}$-range (3-10) and autocitrullinates at amino acid position 63 the arginine to citrulline. Moreover mPPAd citrullinates major autoantigens in RA (Fibrinogen, Vimentin and hnRNP-A2/B1) which are detectable by RA patient sera and specific anti-citrulline monoclonal antibodies. mPPAD citrullinates HeLa-protein extracts and these specific citrullinated proteins are recognized by RA patient sera. Anti-citrullinated mPPAD antibodies were detected in $41 \%(n=32)$ of patients with RA but not in SLE $(n=16)$ and control sera $(n=15)$. In a RA follow-up study $(n=30)$, we detected nearly similar antibody-sensitivities for citrullinated mPPAD before and after onset of RA $(13 / 20 \%)$. Only a minority $(7 \%)$ of RA patients show higher mPPAD antibody levels after RA diagnosis. In the Collagen antibody-induced arthritis (CAIA) RA mouse model mPPAD containing P.g. vesicles when injected IP showed a TLR2-dependent protective anti- inflammatory effect like P.g. LPS and Lipomannan.

Conclusions: Pg. infection and RA disease diagnosis occurs on different timepoints and $\mathrm{Pg}$. infection induces a TLR2-dependent protective anti-inflammatory effect. We show the first time that mPPAD can citrullinate major human autoantigens internally and their immunologically and diagnostic relevance.

Disclosure of Interest: None declared

DOI: 10.1136/annrheumdis-2017-eular.365 